\title{
COMPARATIVE ANALYSIS OF MECHANICAL AND MANUAL MODES OF TRAFFIC SURVEY FOR TRAFFIC LOAD DETERMINATION
}

\author{
K. 0. Kusimo ${ }^{1, *}$ and F. 0. Okafor ${ }^{2}$

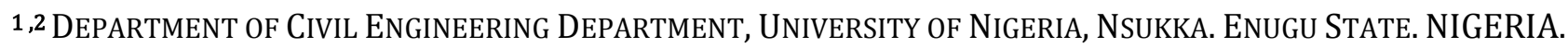 \\ E-mail address: ${ }^{1}$ okusimo@yahoo.com,2fidelis.okafor@unn.edu.ng
}

\begin{abstract}
Traffic survey was carried out to obtain data that are necessary for traffic study and evaluation. One of the most important characteristics of a traffic stream is its volume which can be defined as the number of vehicles passing through a section of a road per unit time. The road project which is approximately $75 \mathrm{~km}$ in length consists of a number of traversed roads, but the traffic study is focused only on the 6 major intersections. The surveys of traffic were conducted using volume count from 7am to 7pm each day because working at night is very unsafe in this area, by using an automatic traffic recorder (Trax 1 Plus) and road tube installation for collecting accurate data with manual counting for comparison. This study aims to analyze the current road traffic data collection methods comparing both mechanical and manual mode of Traffic Survey simulation - in terms of capabilities and limitations on Nigeria roads using Ogoja (Mbok) Junction and Mfum road in Cross River State as a case study. The tendency of the mechanical counting machine to withstand nonstop24hrs-continuous counting was noted during the exercise compared to manual counting, since security at night cannot be predicted on manual. The design equivalent standard axle load (ESAL) generated from the result shows that Ikom-Cameroon Junction has the highest value with a difference of $\left(0.999 \times 10^{\wedge 6}\right)$ between the mechanical and manual, this value shows a downward effect using manual data for the pavement structural thickness evaluation, this can further degenerate to the structural failure at the earlier stage, tensional fatigue, cracks etc. However, traffic study for the period 2015 to 2035 was considered in the analysis, the equivalent standard axle load (ESAL) generated along the section of the corridor and the pavement thickness shows that automatic counter machine is more accurate than the manual. Nonetheless the mechanical counter is more advantageous compared to the manual counter to this reasons the mechanical counter should therefore be employed during the raw data traffic survey. Manual on the other hand less useful for traffic survey in predicting the feature traffic analysis.
\end{abstract}

Keywords: Volume/Flow, Rate of Flow, Average Daily Traffic (ADT), Annual Average Daily Traffic (AADT), Equivalent Single Axle Load (ESAL).

\section{INTRODUCTION}

Traffic volume studies help agencies to make sound traffic safety, to evaluate the need for appropriate traffic control and geometric improvements performance related decisions based on data collected with high degree of accuracy either by mechanical device or manual means.[1,2,3,4] Basically, Traffic volume studies determine the number of movements and classification of vehicles including rush-hour vehicle counting at Intersections, pedestrian counts, average daily traffic, and annual average daily traffic. There are two methods for collecting traffic volume data: automatic and manual.
Automatic counting methods are used to gather large amounts of traffic data over an extended period of time. Counts are generally collected at 1-hour intervals in 24-hour periods.

The following information can be determined using automatic counts:

- hourly traffic patterns

- daily or seasonal variations

- growth trends

- annual traffic estimates

With manual counting methods, observers go to the site and collect data on location for a specific time interval (generally measured with a stop watch). 
Manual counts are typically used when

- small data samples are required.

- automatic equipment is not available, or the effort and expense of using automated equipment are not justified.

- the count period is less than a day.

Manual counts are typically used to gather data about the following:

- vehicle classifications

- turning movements

- direction of travel

- pedestrian movements

- vehicle occupancy

Traffic counts can also be taken at 15-minute interval during the peak hour to determine the peak-hour factor (PHF)'.

The relationship between the peak 15-minute flow rate and the full hourly volume is given by the peakhour factor (PHF) as shown in the following equation:

$$
\mathrm{PHF}=\frac{\text { Hourly volume }}{\text { peak rate of flow within the hour }}
$$

If 15-minute periods are used, the PHF is computed

as:

$$
\text { PHF }=\frac{\mathrm{V}}{\left[4 \times \mathrm{V}_{15}\right]}
$$

Where $\mathrm{V}$ is the peak-hour volume (vph), V15 is the volume during the peak 15 minutes of flow (veh/15 minutes)

\section{MATERIALS AND METHODS}

The road Project traverses four (4) Local Government areas namely; Yala, Ogoja, Ikom, Etung and other important settlements in Cross River State. The beginning of the project at Mbokis geographically located within 6024'.00" North,8042'.00" East and Ikom which is the End of the project is geographically located within 558'.00" North, 842'.00" East. The population of the Project area consists predominantly of farmers cultivating rice, corn, yam, and cassava with petty trading and wood logging at Ikom.

The traffic data collection in the field is done manually and automatically simultaneously, using Automatic Traffic Recorders (ATR) to collect traffic volumes, Automatic Vehicle Classifiers (AVC) to collect the vehicle volumes by vehicle category.

The designed program called TraxPro software was used to process automatic traffic data collected with the TRAX 1 Plus automatic traffic recorders/classifier. The "Traffic counter carried out in the year 2013" will be used as the control data between Trax1 plus data and manual counting for comparison between mechanical and manual counter conducted in 2015.

A continuous count of seven (7) days at $12 \mathrm{hrs}$ count was undertaken by the survey team at each junction to arrive at a more accurate traffic data.

The amount of vehicles to pass during the studies time period, in a lane by lane basis, is determined using 15min. of interval (15-min. interval was adopted in the study).

Various methods can be adopted for verification such as listed below [2]. Combinations of pavement materials and layer thicknesses that will provide satisfactory services over the design life, in this report Overseas Road Note 31was adopted.

a. Overseas Road Note 31 (Updated Version, 1993)

b. Road Note 29 (Old Version)

c. AASHTO Design Guide

\subsection{Overseas Road Note 31}

The Road Note 31 gives recommendations for the structural design of bituminous surfaced roads. It aimed at highway engineers responsible for the design and construction of new road pavements and is appropriate for roads which are required to carry up to 30 million cumulative equivalent axles in one direction.

Using this method, the pavement thicknesses will be determined by using the provided Structural Catalogue and the Design Chart.

\section{RESULTS AND ANALYSIS}

A summary on the Traffic Report are presented in the following Tables:

1. Table 1 to 2: Summary Report on the Number of Vehicles Measured in the Study

2. Table 3: 12-Hour Average Daily Traffic

3. Table 4:24-Hour Average Daily Traffic

4. Table 5: Projected Traffic on Completion of Project and Design Lifespan

5. Table 6: Computation of Design ESAL

The data used as a control for comparative analysis between the mechanical and manual mode of traffic survey was collected in the year 2013, the ESAL generated there was quite difference from the present ESAL due to the following reasons:

1. The number of vehicle diverted to the new facilities has increased due to improvement on the road.

2. Smooth riding surface and

3. Travel time has drastically reduced. 
Comparative Analysis Of Mechanical and Manual Modes of Traffic Survey for ... K. O. Kusimo \& F. o. Okafor

Table 1: Summary report on the number of vehicles measured at Ogoja - Mbok and Edor junctions

\begin{tabular}{|c|c|c|c|c|c|c|c|c|c|c|}
\hline \multicolumn{11}{|c|}{ OGOJA - MBOK JUNCTION } \\
\hline \multirow{2}{*}{ Lane } & \multirow{2}{*}{ Date } & \multicolumn{2}{|c|}{ Time } & \multirow{2}{*}{ Cars } & \multirow{2}{*}{ Buses } & \multirow{2}{*}{$\frac{\text { 2-Axle/4-Tire }}{\text { Trucks }}$} & \multirow{2}{*}{$\frac{\text { 2-Axle/6-Tire }}{\text { Trucks }}$} & \multirow{2}{*}{$\frac{3+\text { Axle Trucks/ }}{\text { Single }}$} & \multirow{2}{*}{$\frac{5+\text { Axle Trucks/ }}{\text { Single }}$} & \multirow{2}{*}{ Tota } \\
\hline & & Start & End & & & & & & & \\
\hline \multirow{7}{*}{1} & $2 / 19 / 2015$ & $9: 28 \mathrm{am}$ & $9: 37: 52 \mathrm{pm}$ & 749 & 1 & 7 & 15 & 24 & 4 & 800 \\
\hline & 2/20/2015 & $8: 36 \mathrm{am}$ & 8:48:16 pm & 1256 & 16 & 23 & 35 & 34 & 50 & 1413 \\
\hline & $2 / 21 / 2015$ & $8: 24 \mathrm{am}$ & 8:34:08 pm & 954 & 5 & 11 & 40 & 16 & 27 & 1054 \\
\hline & $2 / 22 / 2015$ & $8: 32 \mathrm{am}$ & 8:36:00 pm & 1100 & 6 & 16 & 55 & 26 & 40 & 1243 \\
\hline & $2 / 23 / 2015$ & 8:08 am & 8:48:00 pm & 1195 & 0 & 13 & 41 & 23 & 24 & 1295 \\
\hline & $2 / 24 / 2015$ & $8: 52 \mathrm{am}$ & $8: 43: 12 \mathrm{pm}$ & 1344 & 7 & 10 & 42 & 12 & 28 & 1442 \\
\hline & $2 / 25 / 2015$ & $8: 24 \mathrm{am}$ & $8: 35: 28 \mathrm{pm}$ & 1176 & 6 & 17 & 40 & 22 & 16 & 1277 \\
\hline Total Average & & & & 1111 & 6 & 14 & 38 & 22 & 27 & 1218 \\
\hline \multicolumn{11}{|c|}{ EDOR JUNCTION } \\
\hline \multirow{2}{*}{ Lane } & \multirow{2}{*}{ Date } & \multicolumn{2}{|c|}{ Time } & \multirow{2}{*}{ Cars } & \multirow{2}{*}{ Buses } & 2-Axle/4-Tire & 2-Axle/6-Tire & 3+ Axle Trucks/ & 5+ Axle Trucks/ & Tatal \\
\hline & & Start & End & & & Trucks & Trucks & Single & Single & Tulan \\
\hline \multirow{7}{*}{1} & $2 / 19 / 2015$ & $9: 16 \mathrm{am}$ & $9: 42: 24 \mathrm{pm}$ & 1076 & 3 & 5 & 32 & 8 & 40 & 1164 \\
\hline & $2 / 20 / 2015$ & $8: 40 \mathrm{am}$ & 8:53:44 pm & 1575 & 0 & 17 & 40 & 3 & 63 & 1698 \\
\hline & $2 / 21 / 2015$ & 8:44 am & 8:48:32 pm & 1325 & 11 & 29 & 26 & 9 & 51 & 1451 \\
\hline & $2 / 22 / 2015$ & 8:00 am & 8:03:52 pm & 1249 & 9 & 20 & 29 & 8 & 48 & 1364 \\
\hline & 2/23/2015 & $7: 28 \mathrm{am}$ & $7: 35: 20 \mathrm{pm}$ & 1233 & 5 & 8 & 16 & 10 & 47 & 1320 \\
\hline & $2 / 24 / 2015$ & $7: 36 \mathrm{am}$ & $7: 50: 40 \mathrm{pm}$ & 1295 & 6 & 11 & 34 & 12 & 59 & 1418 \\
\hline & $2 / 25 / 2015$ & $8: 24 \mathrm{am}$ & 8:44:48 pm & 1149 & 6 & 15 & 25 & 7 & 44 & 1247 \\
\hline Total Average & & & & 1272 & 6 & 15 & 29 & 8 & 50 & 1380 \\
\hline
\end{tabular}

Table 1a: Summary report on the number of vehicles measured at Four Corner junction

\begin{tabular}{|c|c|c|c|c|c|c|c|c|c|c|}
\hline \multicolumn{11}{|c|}{ FOUR CORNER JUNCTION: } \\
\hline \multirow{2}{*}{ Lane } & \multirow{2}{*}{ Date } & \multicolumn{2}{|c|}{ Date } & \multirow{2}{*}{ Cars } & \multirow{2}{*}{ Buses } & \multirow{2}{*}{$\begin{array}{c}\text { 2-Axle/4-Tire } \\
\text { Trucks }\end{array}$} & \multirow{2}{*}{$\begin{array}{c}\text { 2-Axle/6-Tire } \\
\text { Trucks }\end{array}$} & \multirow{2}{*}{$\begin{array}{c}3+\text { Axle Trucks/ } \\
\text { Single }\end{array}$} & \multirow{2}{*}{$\begin{array}{c}5+\text { Axle Trucks/ } \\
\text { Single }\end{array}$} & \multirow{2}{*}{ Total } \\
\hline & & Start & End & & & & & & & \\
\hline \multirow{7}{*}{1} & $2 / 19 / 2015$ & 8:16 am & 8:39:20pm & 5241 & 211 & 173 & 174 & 64 & 58 & 5920 \\
\hline & $2 / 20 / 2015$ & 7:12 am & $7: 27: 20 \mathrm{pm}$ & 5191 & 277 & 242 & 131 & 109 & 131 & 6082 \\
\hline & $2 / 21 / 2015$ & 7:04 am & $7: 20: 40 \mathrm{pm}$ & 5439 & 279 & 253 & 161 & 114 & 82 & 6327 \\
\hline & $2 / 22 / 2015$ & 8:00 am & 8:08:24 pm & 5333 & 244 & 192 & 146 & 73 & 85 & 6073 \\
\hline & $2 / 23 / 2015$ & $6: 52 \mathrm{am}$ & 7:23:36 pm & 5732 & 309 & 274 & 201 & 92 & 86 & 6694 \\
\hline & $2 / 24 / 2015$ & $6: 40 \mathrm{am}$ & $6: 46: 24 \mathrm{pm}$ & 5738 & 370 & 321 & 196 & 110 & 141 & 6876 \\
\hline & $2 / 25 / 2015$ & 7:12 am & 7:18:16 pm & 5708 & 362 & 278 & 128 & 128 & 151 & 6755 \\
\hline Total Average & & & & 5483 & 293 & 247 & 162 & 99 & 105 & 6389 \\
\hline
\end{tabular}

Table 2 - Summary report on the number of vehicles measured at Ikom - Cameroun and Effraya junctions

\begin{tabular}{|c|c|c|c|c|c|c|c|c|c|c|}
\hline \multicolumn{11}{|c|}{ IKOM - CAMEROUN JUNCTION } \\
\hline \multirow{2}{*}{ Lane } & \multirow{2}{*}{ Date } & \multicolumn{2}{|c|}{ Time } & \multirow{2}{*}{ Cars } & \multirow{2}{*}{ Buses } & \multirow{2}{*}{$\begin{array}{c}\text { 2-Axle/4- } \\
\text { Tire } \\
\text { Trucks }\end{array}$} & \multirow{2}{*}{$\begin{array}{c}\text { 2-Axle/6- } \\
\text { Tire } \\
\text { Trucks }\end{array}$} & \multirow{2}{*}{$\begin{array}{c}3+\text { Axle } \\
\text { Trucks/ } \\
\text { Single }\end{array}$} & \multirow{2}{*}{$\begin{array}{c}5+\text { Axle } \\
\text { Trucks/ } \\
\text { Single }\end{array}$} & \multirow{2}{*}{ Total } \\
\hline & & Start & End & & & & & & & \\
\hline \multirow{5}{*}{1} & $2 / 26 / 2015$ & $6: 44 \mathrm{am}$ & $6: 56: 32 \mathrm{pm}$ & 8813 & 2640 & 232 & 232 & 122 & 183 & 12223 \\
\hline & $2 / 27 / 2015$ & 7:00 am & $6: 59: 44 \mathrm{pm}$ & 10206 & 3058 & 269 & 269 & 142 & 212 & 14155 \\
\hline & $2 / 28 / 2015$ & 6:44 am & $7: 00: 16 \mathrm{pm}$ & 10809 & 3238 & 285 & 285 & 150 & 225 & 14992 \\
\hline & $3 / 1 / 2015$ & 7:16 am & $7: 49: 36 \mathrm{pm}$ & 9466 & 2836 & 249 & 249 & 131 & 197 & 13128 \\
\hline & $3 / 2 / 2015$ & 7:00 am & 7:11:20 pm & 11403 & 3416 & 301 & 301 & 158 & 237 & 15816 \\
\hline
\end{tabular}


Comparative Analysis Of Mechanical and Manual Modes of Traffic Survey for ... K. O. Kusimo \& F. o. Okafor

\begin{tabular}{|c|c|c|c|c|c|c|c|c|c|c|}
\hline & $\begin{array}{l}3 / 3 / 2015 \\
3 / 4 / 2015\end{array}$ & $\begin{array}{l}6: 44 \mathrm{am} \\
7: 24 \mathrm{am}\end{array}$ & $\begin{array}{l}\text { 6:45:04 pm } \\
7: 49: 44 \mathrm{pm}\end{array}$ & $\begin{array}{l}10834 \\
11026\end{array}$ & $\begin{array}{l}3246 \\
3303\end{array}$ & $\begin{array}{l}286 \\
291\end{array}$ & $\begin{array}{l}286 \\
291\end{array}$ & $\begin{array}{l}150 \\
153\end{array}$ & $\begin{array}{l}225 \\
229\end{array}$ & $\begin{array}{l}15027 \\
15292\end{array}$ \\
\hline Total Average & & & & 10365 & 3105 & 273 & 273 & 144 & 216 & 14376 \\
\hline \multicolumn{11}{|c|}{ EFFRAYA JUNCTION: } \\
\hline \multirow{2}{*}{ Lane } & \multirow{2}{*}{ Date } & \multicolumn{2}{|c|}{ Time } & \multirow{2}{*}{ Cars } & \multirow{2}{*}{ Buses } & \multirow{2}{*}{$\begin{array}{c}\text { 2-Axle/4- } \\
\text { Tire } \\
\text { Trucks }\end{array}$} & \multirow{2}{*}{$\begin{array}{c}\text { 2-Axle/6- } \\
\text { Tire } \\
\text { Trucks }\end{array}$} & \multirow{2}{*}{$\begin{array}{l}3+\text { Axle } \\
\text { Trucks/ } \\
\text { Single }\end{array}$} & \multirow{2}{*}{$\begin{array}{c}5+\text { Axle } \\
\text { Trucks/ } \\
\text { Single }\end{array}$} & \\
\hline & & Start & End & & & & & & & \\
\hline \multirow{7}{*}{1} & $2 / 26 / 2015$ & $8: 08 \mathrm{am}$ & 8:29:04 pm & 935 & 138 & 23 & 24 & 13 & 20 & 1152 \\
\hline & 2/27/2015 & 7:44 am & $7: 52: 00 \mathrm{pm}$ & 1052 & 156 & 26 & 27 & 14 & 22 & 1297 \\
\hline & $2 / 28 / 2015$ & $7: 20 \mathrm{am}$ & 7:46:08 pm & 802 & 119 & 20 & 21 & 11 & 17 & 989 \\
\hline & $3 / 1 / 2015$ & $7: 00 \mathrm{am}$ & $7: 12: 48$ pm & 985 & 146 & 24 & 25 & 13 & 21 & 1215 \\
\hline & $3 / 2 / 2015$ & $7: 12 \mathrm{am}$ & 7:21:04 pm & 904 & 134 & 22 & 23 & 12 & 19 & 1114 \\
\hline & $3 / 3 / 2015$ & $8: 00 \mathrm{am}$ & 8:07:44 pm & 1106 & 163 & 27 & 29 & 15 & 23 & 1364 \\
\hline & $3 / 4 / 2015$ & $7: 20 \mathrm{am}$ & 7:31:16 pm & 1068 & 158 & 26 & 28 & 14 & 22 & 1317 \\
\hline $\begin{array}{l}\text { Total } \\
\text { Average } \\
\end{array}$ & & & & 979 & 145 & 24 & 25 & 13 & 20 & 1207 \\
\hline
\end{tabular}

Table 2a -Summary report on the number of vehicles measured at Mfum bridge

\begin{tabular}{|c|c|c|c|c|c|c|c|c|c|c|}
\hline \multicolumn{11}{|c|}{ MFUM BRIDGE } \\
\hline \multirow[t]{2}{*}{ Lane } & \multirow[t]{2}{*}{ Date } & \multicolumn{2}{|c|}{ Time } & \multirow[t]{2}{*}{ Cars } & \multirow[t]{2}{*}{ Buses } & \multirow{2}{*}{$\begin{array}{c}\text { 2-Axle/4- } \\
\text { Tire } \\
\text { Trucks }\end{array}$} & \multirow{2}{*}{$\begin{array}{c}\text { 2-Axle/6- } \\
\text { Tire } \\
\text { Trucks }\end{array}$} & \multirow{2}{*}{$\begin{array}{c}3+\text { Axle } \\
\text { Trucks/ } \\
\text { Single } \\
\end{array}$} & \multirow{2}{*}{$\begin{array}{l}5+\text { Axle } \\
\text { Trucks/ } \\
\text { Single }\end{array}$} & \multirow[t]{2}{*}{ Total } \\
\hline & & Start & End & & & & & & & \\
\hline \multirow{7}{*}{1} & $2 / 26 / 2015$ & $8: 52 \mathrm{am}$ & 9:03:36 pm & 414 & 0 & 2 & 2 & 2 & 0 & 419 \\
\hline & $2 / 27 / 2015$ & $7: 48 \mathrm{am}$ & 8:14:24 pm & 430 & 0 & 0 & 2 & 2 & 0 & 435 \\
\hline & $2 / 28 / 2015$ & $7: 36 \mathrm{am}$ & $7: 47: 36 \mathrm{pm}$ & 381 & 0 & 0 & 0 & 0 & 0 & 381 \\
\hline & $3 / 1 / 2015$ & $7: 28 \mathrm{am}$ & 7:35:44 pm & 439 & 0 & 2 & 0 & 0 & 0 & 441 \\
\hline & $3 / 2 / 2015$ & 8:16 am & 8:38:08 pm & 386 & 0 & 0 & 0 & 4 & 0 & 390 \\
\hline & $3 / 3 / 2015$ & $7: 48 \mathrm{am}$ & 8:07:12 pm & 469 & 0 & 0 & 11 & 0 & 0 & 480 \\
\hline & $3 / 4 / 2015$ & $7: 20 \mathrm{am}$ & 7:32:08 pm & 446 & 0 & 0 & 0 & 0 & 0 & 446 \\
\hline $\begin{array}{c}\text { Total } \\
\text { Average }\end{array}$ & & & & 424 & 0 & 1 & 2 & 1 & 0 & 427 \\
\hline
\end{tabular}

Table 3: 12 Hours Average Daily Traffic

\begin{tabular}{|c|c|c|c|c|c|c|c|c|c|}
\hline $\mathrm{S} / \mathrm{N}$ & Location & & Cars & Buses & $\begin{array}{l}\text { 2-Axle/4- } \\
\text { Tire/Trucks }\end{array}$ & $\begin{array}{l}\text { 2-Axle/6- } \\
\text { Tire/Trucks }\end{array}$ & $\begin{array}{c}\text { 3+ Axle } \\
\text { Trucks/Single }\end{array}$ & $\begin{array}{c}5+\text { Axle } \\
\text { Trucks/Si } \\
\text { ngle }\end{array}$ & Total \\
\hline \multirow{2}{*}{1} & \multirow{2}{*}{$\begin{array}{l}\text { Ogoja - Mbok } \\
\text { Junction }\end{array}$} & ADT & 1111 & 6 & 14 & 38 & 22 & 27 & 1218 \\
\hline & & PCU & 1111 & 12 & 28 & 114 & 66 & 81 & 1412 \\
\hline \multirow{2}{*}{2} & \multirow{2}{*}{ Edor Junction } & ADT & 1272 & 6 & 15 & 29 & 8 & 50 & 1380 \\
\hline & & PCU & 1272 & 11 & 30 & 87 & 24 & 150 & 1574 \\
\hline \multirow{2}{*}{3} & \multirow{2}{*}{$\begin{array}{l}\text { Four Corner } \\
\text { Junction }\end{array}$} & ADT & 5483 & 293 & 247 & 162 & 99 & 105 & 6389 \\
\hline & & PCU & 5483 & 586 & 494 & 486 & 297 & 315 & 7662 \\
\hline \multirow{2}{*}{4} & \multirow{2}{*}{$\begin{array}{l}\text { Ikom-Cameroon } \\
\text { Junction }\end{array}$} & ADT & 10365 & 3105 & 273 & 273 & 144 & 216 & 14376 \\
\hline & & PCU & 10365 & 6211 & 546 & 819 & 431 & 648 & 19021 \\
\hline \multirow{2}{*}{5} & \multirow{2}{*}{ Effraya Junction } & ADT & 979 & 145 & 24 & 25 & 13 & 20 & 1207 \\
\hline & & PCU & 979 & 288 & 47 & 75 & 40 & 61 & 1491 \\
\hline \multirow{2}{*}{6} & \multirow{2}{*}{ Mfum Bridge } & ADT & 424 & 0 & 1 & 2 & 1 & 0 & 427 \\
\hline & & PCU & 424 & 0 & 2 & 6 & 3 & 0 & 435 \\
\hline
\end{tabular}

Passenger Car Unit (PCU) Calculations:

Type of Vehicle PCU Value

Cars \& Taxis $\quad 1.0$

2 Axle vehicles and buses $\quad 2.0$

Heavy duty vehicles $\quad 3.0$ 
Table 4: 24 Hours Average Daily Traffic

\begin{tabular}{|c|c|c|c|c|c|c|c|c|c|}
\hline$S / N$ & Location & & Cars & Buses & $\begin{array}{c}\text { 2-Axle/4- } \\
\text { Tire } \\
\text { Truck }\end{array}$ & $\begin{array}{c}\text { 2-Axle/6- } \\
\text { Tire } \\
\text { Truck }\end{array}$ & $\begin{array}{l}\text { 3+ Axle } \\
\text { Trucks/ } \\
\text { Single }\end{array}$ & $\begin{array}{c}\text { 5+ Axle } \\
\text { Trucks } \\
\text { Single } \\
\text { Trailer }\end{array}$ & Total \\
\hline \multirow{2}{*}{1} & \multirow{2}{*}{$\begin{array}{l}\text { Ogoja - Mbok } \\
\text { Junction }\end{array}$} & ADT & 1521 & 9 & 21 & 59 & 35 & 42 & 1688 \\
\hline & & PCU & 1521 & 16 & 42 & 177 & 105 & 126 & 1988 \\
\hline \multirow{2}{*}{2} & \multirow{2}{*}{ Edor Junction } & ADT & 1742 & 9 & 23 & 45 & 13 & 78 & 1912 \\
\hline & & PCU & 1742 & 18 & 46 & 135 & 39 & 236 & 2217 \\
\hline \multirow{2}{*}{3} & \multirow{2}{*}{$\begin{array}{l}\text { Four Corner } \\
\text { Junction }\end{array}$} & ADT & 7512 & 454 & 384 & 252 & 153 & 162 & 8918 \\
\hline & & PCU & 7512 & 908 & 768 & 756 & 459 & 486 & 10891 \\
\hline \multirow{2}{*}{4} & \multirow{2}{*}{$\begin{array}{l}\text { Ikom - Cameroon } \\
\text { Junction }\end{array}$} & ADT & 14201 & 4813 & 423 & 423 & 223 & 334 & 20419 \\
\hline & & PCU & 14201 & 9625 & 847 & 1271 & 668 & 1002 & 27614 \\
\hline \multirow{2}{*}{5} & \multirow{2}{*}{ Effraya Junction } & ADT & 1341 & 224 & 37 & 39 & 21 & 32 & 1695 \\
\hline & & PCU & 1341 & 450 & 74 & 117 & 62 & 94 & 2138 \\
\hline \multirow{2}{*}{6} & \multirow{2}{*}{ Mfum Bridge } & ADT & 580 & 0 & 1 & 3 & 2 & 0 & 587 \\
\hline & & PCU & 580 & 0 & 2 & 9 & 4 & 0 & 596 \\
\hline
\end{tabular}

Passenger Car Unit (PCU) Calculations:

Type of Vehicle

Cars \& Taxis

2 Axle vehicles and buses

Heavy duty vehicles

Note: Since traffic in Nigeria during night time is relatively low to what it has at daytime an expansion factor of 1.37 for light vehicles and 1.55 for medium/heavy

Table 5 -Projected traffic on completion of project and design lifespan:

\begin{tabular}{|c|c|c|c|c|c|c|c|c|c|c|}
\hline $\mathrm{S} / \mathrm{N}$ & Location & Year & & Cars & Buses & $\begin{array}{l}\text { 2-Axle/4- } \\
\text { Tire } \\
\text { Trucks }\end{array}$ & $\begin{array}{c}\text { 2-Axle/6- } \\
\text { Tire } \\
\text { Trucks }\end{array}$ & $\begin{array}{c}\text { 3+ Axle } \\
\text { Trucks/ } \\
\text { Single }\end{array}$ & $\begin{array}{l}5+\text { Axle } \\
\text { Trucks } \\
\text { Single } \\
\text { Trailer }\end{array}$ & Total \\
\hline \multirow{2}{*}{1} & \multirow{2}{*}{$\begin{array}{l}\text { Ogoja - Mbok } \\
\text { Junction }\end{array}$} & 2006 & ADT & 1646 & 9 & 23 & 64 & 37 & 45 & 1825 \\
\hline & & 2026 & ADT & 3606 & 21 & 50 & 141 & 82 & 100 & 3999 \\
\hline \multirow{2}{*}{2} & \multirow{2}{*}{ Edor Junction } & 2006 & ADT & 1884 & 10 & 25 & 49 & 14 & 85 & 2067 \\
\hline & & 2026 & ADT & 4129 & 22 & 56 & 106 & 30 & 185 & 4528 \\
\hline \multirow{2}{*}{3} & \multirow{2}{*}{$\begin{array}{l}\text { Four Corner } \\
\text { Junction }\end{array}$} & 2006 & ADT & 8125 & 491 & 415 & 272 & 165 & 176 & 9644 \\
\hline & & 2026 & ADT & 17803 & 1076 & 909 & 597 & 362 & 385 & 21132 \\
\hline \multirow[t]{2}{*}{4} & \multirow{2}{*}{$\begin{array}{l}\text { Ikom - } \\
\text { Cameroon } \\
\text { Junction }\end{array}$} & 2006 & ADT & 15359 & 5206 & 458 & 458 & 241 & 362 & 22084 \\
\hline & & 2026 & ADT & 33654 & 11407 & 1003 & 1003 & 528 & 792 & 48388 \\
\hline \multirow{2}{*}{5} & \multirow{2}{*}{$\begin{array}{l}\text { Effraya } \\
\text { Junction }\end{array}$} & 2006 & ADT & 1451 & 243 & 40 & 42 & 22 & 34 & 1833 \\
\hline & & 2026 & ADT & 3179 & 531 & 89 & 93 & 49 & 75 & 4016 \\
\hline \multirow{2}{*}{6} & \multirow{2}{*}{$\begin{array}{l}\text { Mfum - } \\
\text { Cameroon }\end{array}$} & 2006 & ADT & 628 & 0 & 1 & 4 & 2 & 0 & 634 \\
\hline & & 2026 & ADT & 1375 & 0 & 2 & 8 & 4 & 0 & 1389 \\
\hline
\end{tabular}

Projected traffic $=A(1+i)^{n}$, where: $A=$ initial $A D T, i=$ growth rate, $n=$ number of years

Note: Since no data were obtained from the previous traffic count, an assumption in average value of $4 \%$ per year growth rate be taken into consideration.

\section{DISCUSSION OF RESULTS}

The tendency of the mechanical counting machine to withstand non-stop24hrs continuous counting was a major finding during the exercise which has a better advantage compare to manual counting, since security at night cannot be predicted on manual. The design ESAL generated from the comparative shows that Ikom-Cameroon Junction has the highest value of ESAL with a difference of $0.999 \times 10^{\wedge} 6$ between the mechanical and manual, this value shows a 
downward effect using manual data for the pavement structural thickness evaluation, this can further degenerated to the structural failure at the earlier stage, tensional fatigue, cracks etc.

From the impact loading graph generated see Figure $3-5$, it shows that lane2is receiving more loading in terms of volume of the traffic than lane 1 this impact needs to be addressed during the design stage of the structural pavement thickness.

Hence, the relevance of mechanical counting as against the manual cannot be overemphasized. The traffic characteristic are determined in terms of the number of repetition of $80 \mathrm{kN}$ single axle-load applied to the pavement on two sets of dual tires which referred to Equivalent Single Axle Load (ESAL).

The impact on the pavement of the different types of vehicles expected to use the proposed road is obtained from the results of axle classification counts. The 2015 traffic flows on the project road were calculated from the traffic count data collected.

\section{ADVANTAGES AND DISADVANTAGES USING MECHANICAL AND MANUAL COUNTING}

1. Mechanically is more advantageous less stressful, high accuracy compare to manual counter.

2. Maintenance cost is high using mechanical counter compare to manual in terms of procurement, maintenance etc.

3. Safety precaution is better using Mechanical than manual counter.

4. During raining season mechanical cannot be put to use

Table 6: Computation of Design ESAL

\begin{tabular}{|c|c|c|c|c|c|c|c|c|c|c|}
\hline \multirow[b]{2}{*}{$\begin{array}{l}\mathrm{S} / \\
\mathrm{N}\end{array}$} & \multirow[b]{2}{*}{ Location } & \multirow{2}{*}{$\begin{array}{l}\text { INITIAL } \\
\text { ADT }\end{array}$} & \multicolumn{6}{|c|}{ Design ESAL in both direction } & \multirow{2}{*}{$\begin{array}{c}\text { Total } \\
\text { Design ESAL in } \\
\text { both direction }\end{array}$} & \multirow{2}{*}{$\begin{array}{c}\text { Total } \\
\text { Design ESAL in } \\
\text { one direction }\end{array}$} \\
\hline & & & Cars & Buses & $\begin{array}{l}\text { 2-Axle/4- } \\
\text { Tire } \\
\text { Trucks }\end{array}$ & $\begin{array}{c}\text { 2-Axle/6-Tire } \\
\text { Trucks }\end{array}$ & $\begin{array}{c}3+\text { Axle Trucks } / \\
\text { Single }\end{array}$ & $\begin{array}{c}\text { 5+ Axle Trucks } \\
\text { Single Trailer }\end{array}$ & & \\
\hline & $\begin{array}{c}\text { ESAL } \\
\text { FACTOR }\end{array}$ & & 0.0002 & 0.500 & 0.004 & 0.300 & 0.480 & 1.200 & & \\
\hline 1 & $\begin{array}{l}\text { Ogoja- } \\
\text { Mbok } \\
\text { Junction }\end{array}$ & 1,646 & 3,578 & 48,914 & 1,000 & 208,698 & 193,046 & 586,964 & $1,042,200$ & 521,100 \\
\hline 2 & $\begin{array}{l}\text { Edor } \\
\text { Junction }\end{array}$ & 1,884 & 4,096 & 54,349 & 1,087 & 159,785 & 73,044 & $1,108,709$ & $1,401,070$ & 700,535 \\
\hline 3 & $\begin{array}{l}\text { Four } \\
\text { Corner } \\
\text { Junction }\end{array}$ & 8,125 & 17,663 & $2,668,511$ & 18,044 & 886,968 & 860,880 & $2,295,681$ & $6,747,747$ & $3,373,873$ \\
\hline 4 & $\begin{array}{l}\text { Ikom - } \\
\text { Cameroon } \\
\text { Junction }\end{array}$ & 15,359 & 33,390 & $28,293,829$ & 19,913 & $1,493,497$ & $1,257,407$ & $4,721,798$ & $35,819,833$ & $17,909,917$ \\
\hline 5 & $\begin{array}{l}\text { Effraya } \\
\text { Junction }\end{array}$ & 1,451 & 3,154 & $1,320,669$ & 1,739 & 136,958 & 114,784 & 443,484 & $2,020,788$ & $1,010,394$ \\
\hline 6 & $\begin{array}{l}\text { Mfum } \\
\text { Bridge }\end{array}$ & 628 & 1,365 & 0 & 43 & 13,044 & 10,435 & 0 & 24,887 & 12,444 \\
\hline
\end{tabular}

Table 7 - Summary of Design ESAL values for each section:

\begin{tabular}{cclccc}
\hline \multirow{2}{*}{$\mathrm{S} / \mathrm{N}$} & \multirow{2}{*}{ Section Limits } & \multicolumn{3}{c}{ Design ESAL Accuracy } & \multirow{2}{*}{ ESAL, Different } \\
\cline { 3 - 5 } & & Trax 1 Plus, 2015 & Manual, 2015 & Manual Traffic count, 2013 & \\
\hline 1 & $\mathrm{~km} 00+000$ to $30+000$ & $0.521 \times 10^{\wedge 6}$ & $0.405 \times 10^{\wedge 6}$ & $0.370 \times 10^{\wedge 6}$ & $0.151 \times 10^{\wedge 6}$ \\
\hline 2 & $\mathrm{~km} 30+500$ to $45+000$ & $0.700 \times 10^{\wedge 6}$ & $0.69 \times 10^{\wedge 6}$ & $0.512 \times 10^{\wedge 6}$ & $0.188 \times 10^{\wedge 6}$ \\
\hline 3 & $\mathrm{~km} 45+500$ to $75+000$ & $3.374 \times 10^{\wedge 6}$ & $3.00 \times 10^{\wedge 6}$ & $2.90 \times 10^{\wedge 6}$ & $0.474 \times 10^{\wedge 6}$ \\
\hline 4 & $\mathrm{~km} 52+400$ to $61+400$ & $17.910 \times 10^{\wedge 6}$ & $17.54 \times 10^{\wedge 6}$ & $16.911 \times 10^{\wedge 6}$ & $0.999 \times 10^{\wedge 6}$ \\
\hline 5 & $\mathrm{~km} 61+400$ to $75+000$ & $1.010 \times 10^{\wedge 6}$ & $0.99 \times 10^{\wedge 6}$ & $0.95 \times 10^{\wedge 6}$ & $0.06 \times 10^{\wedge 6}$ \\
\hline
\end{tabular}




\section{CONCLUSION}

The fundamental differences between mechanical and manual mode of traffic survey in which the results are based on data collected, 6-series of analysis runs were performed for every test and scenario and an average collected, it only reduces the average error and gets the results closer to the true convergence value of the computation.

In general, the following conclusions could be drawn from the basis of the analysis

1. Manual is not sensitive to various geometric factors such as lane width, length of acceleration lane, length of deceleration lane etc.

2. It was also found that manual results compared fairly well with mechanical mode but the results did not match.

3. Error due to human during the data collection is a major factor on the side of the manual counting

4. Automatic survey is used for collection of long hour traffic survey.

5. The resultant effect of the ESAL value when wrongly computed downward effect on the selection of pavement structure as follows: structural thickness, cracks at the earlier stage, tensional effect etc.

6. As a result of improvement on the facility there is generation of high vehicular movement diverted to the new facility comparing the ESAL generated in the year 2013 with 2015 see Table 7.

7. From the graph generated from the software shows that traffic on lane 2 is impacting higher traffic see Figure 1-3 Volume report graph, in this case attention on pavement thickness should be addressed during design stage.

None the less the mechanical counter has better advantage compare to the manual counter to this reasons the mechanical counter should therefore be employed during the raw data traffic counter. Manual on the other hand less useful for traffic survey in predicting the future traffic analysis.

\section{REFERENCES}

[1] Garber, N. J. and Hoel, L. A. Traffic and Highway Engineering, Cengage Learning, 2010.

[2] Mwakalonge, J. L., Waller, J. C., and Perkins, J. A.Temporal Stability and Transferability of NonMotorized and Total Trip Generation Models',Journal of Transportation Technologies, 2012.http://www.SciRP.org/journal/jtts Accessed on September 21, 2015.

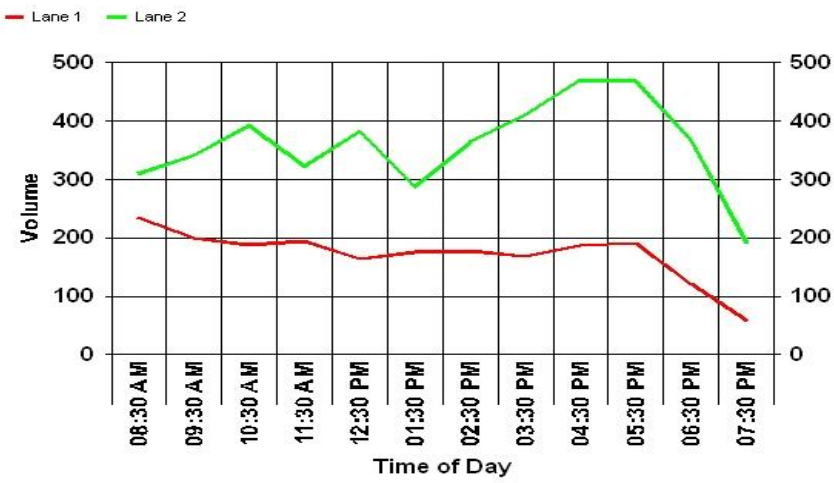

Figure 1: Volume report graph 2-26-15

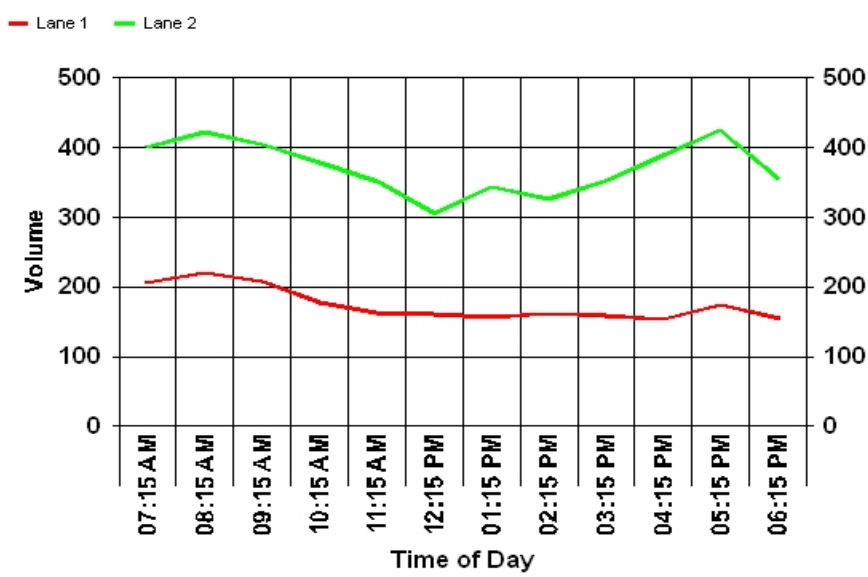

Figure 2: Volume report graph 2-27-15

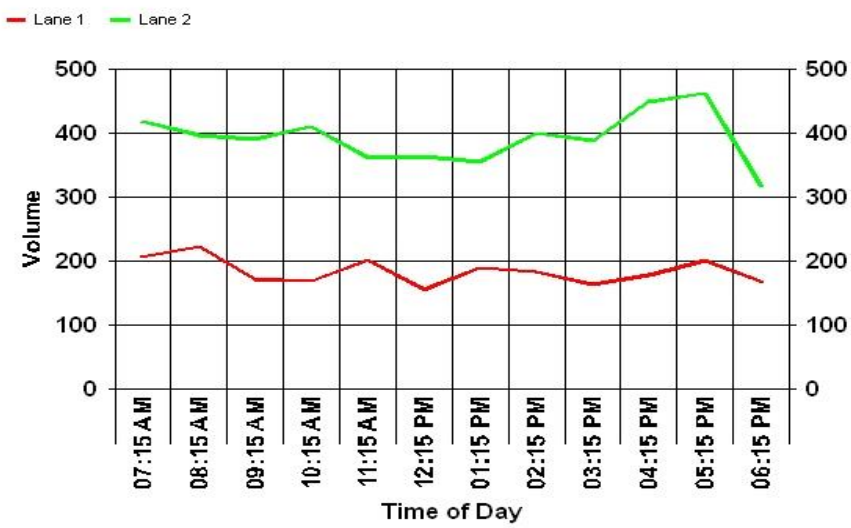

Figure 3: Volume report graph 2-28-15

[3] Adeleke, 0. O., Jimoh, Y. A., Salami, A. W "Determination of Capacity at Traffic Warden Controlled Intersection Using Fixed-Time Signalised Intersection Capacity Model". International Journal of Engineering, Tome XI, Fascicule 3, 2013, pp. 33 36.

[4] O'Flaherty C. A. Transport Planning and Traffic Engineering, Elsevier Ltd., UK, 1997.

[5] Institute of Transportation Engineers. Design and Safety of Pedestrian Facilities, a Recommended 
Practice, Institute of Transportation Engineers, 1998.

[6] Jimoh, Y.A. and Adeleke, 0.0. 'Potential Benefits of Intelligent Transportation System (ITS) in Nigeria', 359 USEP: Journal of Research in Civil Engineering 2 (1) (2005) 46-56.

[7] Naqvi, H.M. Applications of ITS in Bus Transit. National Workshop on Intelligent Transportation System, 361 Pune, India, 25-26 June, 2010.

[8] Vieira, V., Salgado, A.C., Tedesco, P., Times, V., Ferraz, C., Huzita, H., Chaves, A.P., Steinmacher, I. 'The UbiBus 363 Project: Using Context and Ubiquitous Computing to Build Advanced Public Transportation Systems to 364 Support Bus Passengers'.

http://www.cin.ufpe.br/ ubibus/artigos/VieiraEt Al_UBIBUS_SBSI2012.pdf 365 Accessed on February. 24, 2013.

[9] Shankar, K.V.R.R. 'Applications of Advanced Technologies to Transportation Systems in Indian Context', 367 International Journal of Earth Sciences and Engineering 04 (06 SPL), 2011. pp. 394-397.

[10] US Department of Transportation. Advanced Public Transportation Systems: The State of the Art, Update 370 '94, US Department of Transportation, 1994.

[11] World Resource Institute. Modernising Public Transportation: Lessons Learned from Major Bus 372 Improvements in Latin America and Asia, The WRI Center for Sustainable Transport, USA, 2010.

[12] Vanajakshi, L. Ramadurai, G. Anand, A. 'Intelligent Transportation Systems, Synthesis Report on ITS 374 Including Issues and Challenges in India', IIT Madras, India, December 2010. 375
<http://coeut.iitm.ac.in/ITS_synthesis.pdf> Accessed on February 24, 2013.

[13] Adebambo S. and Adebayo, I. T. 'Impact of Bus Rapid Transit System (BRT) On Passengers' Satisfaction in 377 Lagos Metropolis, Nigeria'.

[14] Efimenko, D. B. ITS-related Aspects of Development of Urban Transport Systems. Moscow State Automobile 379 and Road Technical University Department, Russia.

[15] Ben-Akiva, M. Intelligent Transportation Systems (ITS) and the Impact of Traveler Information \& Emerging 381 Themes in Transportation Economics and Policy. Massachusetts Institute of Technology, USA.

[16] U.S. Department of Transportation, Intelligent Transportation Systems Benefits, Costs, Deployment, and 383 Lessons Learned, 2008 Update, U.S. Department of Transportation 2008

[17] U.S. Department of Transportation, The Transportation Planning Process: Key Issues, U.S. Department of 385 Transportation, 2005

[18] Ezell, S. 'Bringing U.S. Roads into the 21st Century'. Issues in Science and Technology 26, no. 4, Summer 3872010.

[19] Elizer, M. 'Strategies and Outlook for ITS Financing'. http://nationalruralitsconference.org/ downloads/Presentations12/B1_Elizer.pdf Accessed February 12, 3902016

[20] European Commission. Study Regarding Guidline for Public Funding of Intelligent Transportation Systems, 392 Final Report, European Commission/ DG MOVE, 2011. 